\title{
Development of General Catalytic Allylation of Acylhydrazones with Pinacolyl Allylboronate Using an Indium(I) Catalyst
}

\author{
Uwe Schneider, I-Hon Chen, Shū Kobayashi* \\ Department of Chemistry, School of Science and Graduate School of Pharmaceutical \\ Sciences, The University of Tokyo, \\ The HFRE Division, ERATO, Japan Science and Technology Agency (JST), \\ Hongo, Bunkyo-ku, Tokyo 113-0033, Japan
}

\section{Supporting Information Part 1:}

\section{General Information}

Table of Contents

Page

General Experimental

S-1

Procedure for Indium(I) lodide-Catalyzed Allylation of 1a-u with 2

$\mathrm{S}-2$

Procedure for Indium(0)-Mediated Barbier-Type Allylation of 1a

S-2

Procedures for NMR Experiments A, B, C and D

S-3

Analytical Data for Homoallylic Hydrazides 3a-u

S-6

References

S-8

\section{General Experimental}

NMR spectra were recorded on a JEOL JNM-ECX400 spectrometer, operating at $400 \mathrm{MHz}$ for ${ }^{1} \mathrm{H}$ NMR, $100 \mathrm{MHz}$ for ${ }^{13} \mathrm{C}$ NMR and $128 \mathrm{MHz}$ for ${ }^{11} \mathrm{~B}$ NMR. Chemical shifts were reported downfield from TMS or in the scale relative to the corresponding solvent used as an internal reference. IR spectra were measured using a JASCO FT/IR-610 spectrometer. ESI high-resolution mass spectra (ESI-HRMS) were measured with BRUKER DALTONICS BioTOF II. Melting points were measured using a BÜCHI B-545 apparatus and are uncorrected. Preparative thin-layer chromatography was carried out using Wakogel B-5F. 
All solvents used in this work were commercially available dry solvents or were distilled under an argon atmosphere. Toluene was freshly distilled from sodium/benzophenone; methanol was distilled from magnesium and stored under argon over molecular sieves $3 \AA$. Acylhydrazones were synthesized according to a literature-known procedure; ${ }^{1}$ prior to their use acylhydrazones were recrystallized and dried ( $80^{\circ} \mathrm{C}$ under HV for several hours). Commercially available pinacolyl allylboronate (2) was prepared by a modified procedure of a known method. ${ }^{2}$ Indium(I) iodide (powder; 99.999\%) was purchased from Aldrich and was used without further purification. All reactions were carried out under an argon atmosphere in well-dried glassware.

\section{Procedure for Indium(I) lodide-Catalyzed Allylation of 1 a-u with 2}

To a dried septum-capped $10 \mathrm{~mL}$-flask with magnetic stirring bar under an argon atmosphere were added indium(l) iodide (4.8 $\mathrm{mg} ; 5 \mathrm{~mol} \%$ ) and the corresponding acylhydrazone 1a-u $(0.4 \mathrm{mmol})$. After addition of dry toluene (800 $\mu \mathrm{L} ; 0.5 \mathrm{M})$ and dry methanol (81 $\mu \mathrm{L} ; 2.0 \mathrm{mmol} ; 5.0$ equiv) was added pinacolyl allylboronate (2; $112.5 \mu \mathrm{L}, 0.6 \mathrm{mmol} ; 1.5$ equiv). The mixture was stirred under an argon atmosphere at room temperature until thin-layer chromatography (TLC) indicated complete conversion of the corresponding hydrazone. After dilution with dichloromethane was added an aqueous solution of $\mathrm{NaHCO}_{3}$ (sat.) and the phases were separated. The aqueous phase was then extracted with dichloromethane (three times) and the combined organic layers were dried $\left(\mathrm{Na}_{2} \mathrm{SO}_{4}\right)$,

filtered and concentrated in vacuo. The residues were purified by preparative thin-layer chromatography (PTLC; eluent: $n$-hexane/ethyl acetate $=9: 1$ to $2: 1$ ) to afford the corresponding homoallylic hydrazides $\mathbf{3 a - u}$.

\section{Procedure for Indium(0)-Mediated Barbier-Type Allylation of 1 a}

To a dried septum-capped $10 \mathrm{~mL}$-flask with magnetic stirring bar under an argon atmosphere were added indium(0) $(68.9 \mathrm{mg} ; 0.6 \mathrm{mmol} ; 1.5$ equiv) and acylhydrazone $1 \mathrm{a}$ (64.9 mg; $0.4 \mathrm{mmol})$. After addition of dry toluene (800 $\mu \mathrm{L} ; 0.5 \mathrm{M})$ and dry methanol (81 $\mu \mathrm{L} ; 5.0$ equiv) was added allyl bromide (51.9 $\mu \mathrm{L}, 0.6 \mathrm{mmol} ; 1.5$ equiv). The mixture was stirred under an argon atmosphere at room temperature for $6 \mathrm{~h}$ (complete conversion 
according to TLC analysis). The work-up procedure was the same as in the indium(I) iodide-catalyzed allylation of acylhydrazones with 2 . The residue was purified by preparative thin-layer chromatography (eluent: $n$-hexane/ethyl acetate $=4: 1$ ) to afford the desired homoallylic hydrazide 3 a (75.6 mg; yield: 93\%)

\section{Procedures for NMR Experiments A, B, C and D}

The authetic sample of pinacol allylboronate (2) in $\mathrm{CDCl}_{3}$ shows four sets of signals at 1.23 ppm (pinacol moiety, 12H), 1.69-1.71 ppm (allylic $\mathrm{CH}_{2}, 2 \mathrm{H}$ ), 4.89-5.00 ppm (vinylic $\mathrm{CH}_{2}$, $2 \mathrm{H}$ ) and 5.78-5.89 ppm (vinylic $\mathrm{CH}, 1 \mathrm{H}$ ) in the ${ }^{1} \mathrm{H}$ NMR chart (Chart $N^{\circ} 1$ ) and one signal at $\sim 32 \mathrm{ppm}$ in the ${ }^{11} \mathrm{~B}$ NMR chart $\left(\right.$ Chart $\left.N^{\circ} 2\right)$. The allylic and vinylic signals $\left({ }^{1} \mathrm{H}\right.$ NMR) as well as the boron signal $\left({ }^{11} \mathrm{~B} N M R\right)$ were used as reference peaks for the NMR experiments (Charts $N^{\circ} 2$ and 3). NMR spectroscopic analyses were conducted at room temperature with an aliquot transferred from the corresponding experiment into a dry septum-capped NMR tube purged with argon.

\section{Experiment A (toluene- $d_{8} /$ methanol- $\left.d_{3}\right)$ :}

${ }^{1} \mathrm{H} N M R$ and ${ }^{11} \mathrm{~B} N M R$ Charts $N^{\circ} 4$ and 5 (5 min), 6 and 7 (30 min), 8 and 9 (3 h)

To a dried septum-capped $3 \mathrm{~mL}$-vial with magnetic stirring bar under an argon atmosphere were added successively indium(I) iodide (100 mol \%), dry toluene- $d_{8}(0.75 \mathrm{M})$, dry methanol- $d_{3}$ (3.33 equiv) and pinacolyl allylboronate $(2 ; 1.0-1.5 \mathrm{mmol} ; 1.0$ equiv). The mixture was stirred under an argon atmosphere at room temperature and was monitored with time (5 min, $30 \mathrm{~min}, 3 \mathrm{~h}$ ) with ${ }^{1} \mathrm{H}$ and ${ }^{11} \mathrm{~B}$ NMR spectroscopic analyses.

In this experiment, even after 5 min two new sets of signals at 1.53-1.55 ppm and $\sim 5.6-5.8$ ppm could already be observed with ${ }^{1} \mathrm{H}$ NMR analysis (Chart $N^{\circ} 4$ ); these peaks show an upfield shift compared with the signals of allylboronate 2 . The intensities of these signals increased with time (Chart $N^{\circ}$ ) and after $3 \mathrm{~h}$ allylboronate 2 was almost completely converted into a single new species, allylindium $(\mathrm{I})^{3}$ (Chart $N^{*}$ 8). This boron-to-indium transmetallation process could be detected as well with ${ }^{11} \mathrm{~B}$ NMR analysis, where the initial signal of 2 at $\sim 32$ ppm progressively disappeared to form two major signals at $\sim 21 \mathrm{ppm}$ 
and $\sim 17 \mathrm{ppm}$ (Charts $N^{\circ}$ 5, 7 and 9). These peaks can be ascribed to borate species such as $\mathrm{B}(\mathrm{pin}) \mathrm{OR}$ and $\mathrm{B}(\mathrm{OR})_{3}$ and show that boron was stripped off the allylic moiety.

In conclusion, a remarkably selective boron-to-indium transmetallation occurred from allylboronate 2 and indium(I) iodide in toluene at room temperature in the presence of methanol.

\section{Experiment B (Barbier-type; toluene- $d_{8} /$ methanol- $d_{3}$ ):}

${ }^{1} \mathrm{H}$ NMR Charts $N^{\circ} 10(5 \mathrm{~min})$ and $11(20 \mathrm{~h})$

To a dried septum-capped $3 \mathrm{~mL}$-vial with magnetic stirring bar under an argon atmosphere were added successively indium(0) $(100 \mathrm{~mol} \%)$, dry toluene- $d_{8}(0.75 \mathrm{M})$, dry methanol- $d_{3}$ (3.33 equiv) and allyl bromide (1.0-1.5 mmol; 1.0 equiv). The mixture was stirred under an argon atmosphere at room temperature and was monitored with time (5 min, $20 \mathrm{~h})$ with ${ }^{1} \mathrm{H}$ NMR spectroscopic analyses.

In this experiment, after 5 min the signals of allyl bromide [3.40-3.42 ppm (allylic $\mathrm{CH}_{2}, 2 \mathrm{H}$ ), 4.73-4.90 ppm (vinylic $\mathrm{CH}_{2}, 2 \mathrm{H}$ ), 5.60-5.71 ppm (vinylic $\mathrm{CH}, 1 \mathrm{H}$ )] could be observed without change of the ${ }^{1} \mathrm{H}$ NMR chart (Chart $N^{0} 10$ ). After overnight stirring, allyl bromide was completely consumed and a single new set of signals with upfield shift compared with the initial peaks of allyl bromide could be observed (Chart $N^{0} 11$ ). These signals at 1.53$1.55 \mathrm{ppm}(2 \mathrm{H}), 4.89-4.98 \mathrm{ppm}(2 \mathrm{H})$ and $5.64-5.73 \mathrm{ppm}(1 \mathrm{H})$ are identical with the ones observed in experiment $\mathrm{A}$ after $3 \mathrm{~h}$ and can be ascribed to allylindium(I) (cf. Chart $N^{\circ} 8$ ).

In conclusion, allylindium(I) could be independently prepared from allyl bromide and indium( $(0)$ under Barbier-type conditions in the same solvent system as in experiment $A$.

\section{Experiment C (toluene- $d_{8}$ ):}

${ }^{1} H$ NMR and ${ }^{11} B$ NMR Charts $N^{\circ} 12$ and 13 (5 min), 14 and 15 (1 h), 16 and 17 (3 h)

To a dried septum-capped $3 \mathrm{~mL}$-vial with magnetic stirring bar under an argon atmosphere were added successively indium(l) iodide $(100 \mathrm{~mol} \%)$, dry toluene- $d_{8}(0.75 \mathrm{M})$ and pinacolyl allylboronate $(2 ; 1.0-1.5 \mathrm{mmol} ; 1.0$ equiv). The mixture was stirred under an 
argon atmosphere at room temperature and was monitored with time ( $5 \mathrm{~min}, 1 \mathrm{~h}, 3 \mathrm{~h})$ with ${ }^{1} \mathrm{H}$ and ${ }^{11} \mathrm{~B}$ NMR spectroscopic analyses.

In this experiment, even after $3 \mathrm{~h}$ no significant change could be detected with ${ }^{1} \mathrm{H}$ NMR analysis (Charts $N^{\circ} 12,14$ and 16). This observation could be confirmed with ${ }^{11} \mathrm{~B}$ NMR analysis, where only a tiny new signal at $\sim 21 \mathrm{ppm}[\mathrm{B}(\mathrm{pin}) \mathrm{OR}]$ appeared (Charts $N^{0} 13,15$ and 17); this peak can be ascribed to a minor decomposition of allylboronate 2 due to traces of humidity.

In conclusion, in the absence of methanol no boron-to-indium transmetallation occurred from allylboronate 2 and indium(I) iodide in toluene at room temperature.

\section{Experiment D (THF- $d_{8}$ ):}

${ }^{1} \mathrm{H} N \mathrm{NMR}$ and ${ }^{11} \mathrm{~B}$ NMR Charts $N^{\circ} 18$ and 19 (5 min), 20 and 21 (1 h) and 22 and 23 (3 h)

To a dried septum-capped $3 \mathrm{~mL}$-vial with magnetic stirring bar under an argon atmosphere were added successively indium(I) iodide (100 mol \%), dry THF- $d_{8}(0.75 \mathrm{M})$ and pinacolyl allylboronate (2; $1.0-1.5 \mathrm{mmol} ; 1.0$ equiv). The mixture was stirred under an argon atmosphere at room temperature and was monitored with time ( $5 \mathrm{~min}, 1 \mathrm{~h}, 3 \mathrm{~h}$ ) with ${ }^{1} \mathrm{H}$ and ${ }^{11}$ B NMR spectroscopic analyses.

In this experiment, a small amount of several new downfield shifted signals appeared with time, detectable with ${ }^{1} \mathrm{H}$ NMR analysis (Charts $N^{0}$ 18, 20 and 22); these peaks at $\sim 1.7 \mathrm{ppm}$, 1.8 ppm and $2.2 \mathrm{ppm}$ can be ascribed to a small amount of different allylindium species. ${ }^{4,5}$ However, the initial allylboronate $\mathbf{2}$ is by far the major compound even after $3 \mathrm{~h}$. This observation could be confirmed with ${ }^{11} \mathrm{~B}$ NMR analysis, where only a small new signal at 21 ppm [B(pin)OR] appeared with time (Charts $N^{\circ} 19,21$ and 23); this peak can be ascribed to a minor boron-to-indium transmetallation.

In conclusion, in the absence of methanol only a sluggish non-selective boron-to-indium transmetallation occurred from allylboronate 2 and indium(I) iodide in THF at room temperature. 


\section{Analytical Data for Homoallylic Hydrazides 3a-u}

Homoallylic hydrazides $\mathbf{3 a - c}, \mathbf{3 e - h}, \mathbf{3} \mathbf{j}-\mathbf{n}$ and $\mathbf{3} \mathbf{s}-\mathbf{t}^{1,6}$ as well as $\mathbf{3} \mathbf{q}^{7}$ are known compounds; obtained analytical data are in agreement with reported data (cf. copies of ${ }^{1} \mathrm{H}$ and ${ }^{13} \mathrm{C}$ NMR spectra).

Analytical data of new homoallylic hydrazides $\mathbf{3 d}, \mathbf{3 i}, \mathbf{3 0 - p}, \mathbf{3 r}$ and $\mathbf{3 u}$ are as follows.

$\boldsymbol{N}$-(1-(1'-tert-butyldimethylsiloxy)hex-5-en-3-yl)benzohydrazide (3d; Table 2, entry

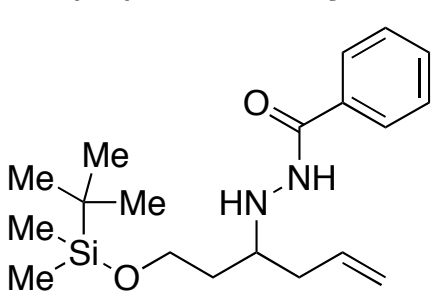

3d

4): prepared from hydrazone 1d according to the general procedure for indium(I) iodide-catalyzed allylation with 2. Yellow oil (yield: 96\%); ${ }^{1} \mathbf{H}$ NMR $\left(\mathrm{CDCl}_{3}, 400 \mathrm{MHz}\right): \delta=0.05(\mathrm{~s}, 6 \mathrm{H}), 0.86$ $(\mathrm{s}, 9 \mathrm{H}), 1.66-1.72(\mathrm{~m}, 2 \mathrm{H}), 2.24(\mathrm{t}, J=6.8 \mathrm{~Hz}, 2 \mathrm{H}), 3.08-3.14(\mathrm{~m}$, $1 \mathrm{H})$, 3.72-3.84 (m, 2H), 5.08-5.15 (m, 2H), 5.22 (br s, $1 \mathrm{H}), 5.80-$ $5.91(\mathrm{~m}, 1 \mathrm{H}), 7.37-7.48(\mathrm{~m}, 3 \mathrm{H}), 7.71-7.73(\mathrm{~m}, 2 \mathrm{H}), 8.19(\mathrm{br} \mathrm{s}$, $1 \mathrm{H}) ;{ }^{13} \mathrm{C} \mathrm{NMR}\left(\mathrm{CDCl}_{3}, 100 \mathrm{MHz}\right): \delta=-5.39,-5.35,18.25,25.89$, $35.93,38.04,58.14,60.99,117.50,126.79,128.52,131.56$, 133.03, 135.20, 166.58; IR (neat): $v=3286,3071,2954,2928,2884,2857,1638,1578$, 1540, 1462, 1360, 1313, 1257, 1095, 1027, 914, 837, 805, 777, 696, 664, $514 \mathrm{~cm}^{-1}$; HRMS (ESI) calcd. for $\mathrm{C}_{19} \mathrm{H}_{33} \mathrm{~N}_{2} \mathrm{O}_{2} \mathrm{Si}^{+}=[\mathrm{M}+\mathrm{H}]^{+}: m / z=349.2306$, found: $m / z=349.2294$.

$\boldsymbol{N}$-(hexa-1,5-dien-3-yl)benzohydrazide (3i; Table 2, entry 9): prepared from

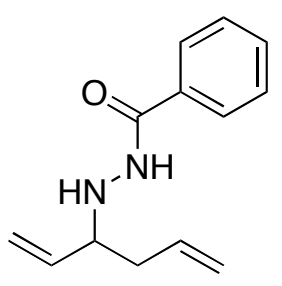

$3 \mathbf{i}$ hydrazone $1 \mathbf{i}$ according to the general procedure for indium(I) iodidecatalyzed allylation with 2 . Pale yellow solid (yield: $82 \%$ ); $\mathrm{mp}=65.4-67.5$ ${ }^{\circ} \mathrm{C} ;{ }^{1} \mathrm{H}$ NMR $\left(\mathrm{CDCl}_{3}, 400 \mathrm{MHz}\right): \delta=2.30(\mathrm{dd}, J=6.8 \mathrm{~Hz}, J=7.2 \mathrm{~Hz}, 2 \mathrm{H})$, $3.54(\mathrm{dt}, J=6.8 \mathrm{~Hz}, J=7.2 \mathrm{~Hz}, 1 \mathrm{H}), 5.00(\mathrm{br} \mathrm{s}, 1 \mathrm{H}), 5.10-5.23(\mathrm{~m}, 4 \mathrm{H})$, 5.66-5.77 (m, 1H), 5.78-5.90 (m, 1H), 7.38-7.52 (m, 3H), $7.61(\mathrm{br} \mathrm{s}, 1 \mathrm{H})$, 7.67-7.72 (m, 2H); ${ }^{13} \mathrm{C}$ NMR $\left(\mathrm{CDCl}_{3}, 100 \mathrm{MHz}\right): \delta=38.06,62.96,117.87$, $118.10,126.78,128.69,131.81,132.89,134.24,138.52,167.03 ;$ IR (neat): $v=3281,3076,2980,2908,1641,1604,1578,1537,1460,1324,1067,1027,992$, 921, 797, $694 \mathrm{~cm}^{-1}$; HRMS (ESI) calcd. for $\mathrm{C}_{13} \mathrm{H}_{17} \mathrm{~N}_{2} \mathrm{O}^{+}=[\mathrm{M}+\mathrm{H}]^{+}: \mathrm{m} / z=217.1335$ and for $\mathrm{C}_{13} \mathrm{H}_{16} \mathrm{~N}_{2} \mathrm{Na}^{+}=[\mathrm{M}+\mathrm{Na}]^{+}: m / z=239.1155$, found: $m / z=217.1339$ (major) and 239.1160 (minor).

$\boldsymbol{N}$-(1-(2-methoxyphenyl)but-3-enyl)benzohydrazide (30; Table 2, entry 15): prepared

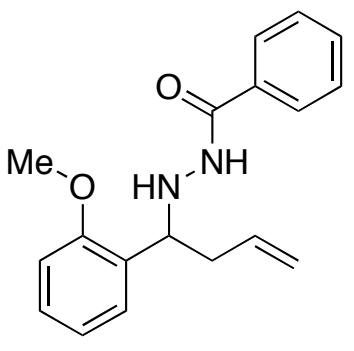

30 from hydrazone 10 according to the general procedure for indium(I) iodide-catalyzed allylation with 2. Pale yellow oil (yield: $98 \%$ ); ${ }^{1} \mathbf{H}$ NMR $\left(\mathrm{CDCl}_{3}, 400 \mathrm{MHz}\right): \delta=2.40-2.50(\mathrm{~m}, 1 \mathrm{H}), 2.51-2.60(\mathrm{~m}, 1 \mathrm{H})$, $3.76(\mathrm{~s}, 3 \mathrm{H}), 4.60(\mathrm{dd}, J=7.6 \mathrm{~Hz}, J=8.0 \mathrm{~Hz}, 1 \mathrm{H}), 5.08-5.19(\mathrm{~m}, 2 \mathrm{H})$, $5.33(\mathrm{br} \mathrm{s}, 1 \mathrm{H}), 5.83-5.95(\mathrm{~m}, 1 \mathrm{H}), 6.86(\mathrm{~d}, J=8.4 \mathrm{~Hz}, 1 \mathrm{H}), 6.97(\mathrm{dd}, J$ $=7.2 \mathrm{~Hz}, J=7.6 \mathrm{~Hz}, 1 \mathrm{H}), 7.21-7.52(\mathrm{~m}, 6 \mathrm{H}), 7.58(\mathrm{dd}, J=1.2 \mathrm{~Hz}, J=$ $7.2 \mathrm{~Hz}, 2 \mathrm{H}) ;{ }^{13} \mathrm{C}$ NMR $\left(\mathrm{CDCl}_{3}, 100 \mathrm{MHz}\right): \delta=38.76,55.34,57.35$, $110.66,117.55,120.62,126.77,127.43,128.28,128.55,129.59$, 131.60, 133.04, 135.14, 157.55, 166.79; IR (neat): $v=3286,3071,2936,2836,1642$, 1602, 1579, 1536, 1492, 1463, 1289, 1243, 1051, 1028, 917, 756, $694 \mathrm{~cm}^{-1}$; HRMS (ESI) 
calcd. for $\mathrm{C}_{18} \mathrm{H}_{21} \mathrm{~N}_{2} \mathrm{O}_{2}^{+}=[\mathrm{M}+\mathrm{H}]^{+}: m / z=297.1598$ and for $\mathrm{C}_{18} \mathrm{H}_{20} \mathrm{~N}_{2} \mathrm{NaO}_{2}{ }^{+}=[\mathrm{M}+\mathrm{Na}]^{+}: \mathrm{m} / z=$ 319.1417, found: $m / z=297.1591$ (major) and 319.1407 (minor).

$\boldsymbol{N}$-(1-(4-(dimethylamino)phenyl)but-3-enyl)benzohydrazide (3p; Table 2, entry 16):

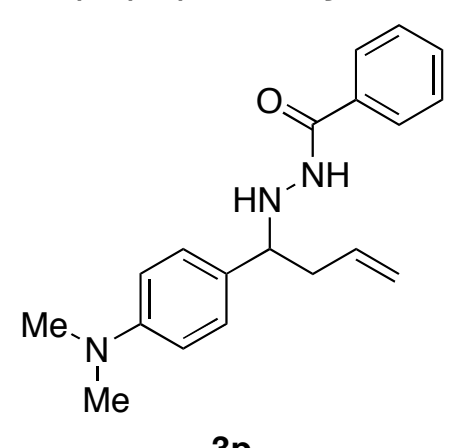

$3 p$ prepared from hydrazone $1 \mathrm{p}$ according to the general procedure for indium(I) iodide-catalyzed allylation with 2 . Pale yellow oil (yield: $75 \%) ;{ }^{1} \mathrm{H}$ NMR $\left(\mathrm{CDCl}_{3}, 400 \mathrm{MHz}\right): \delta=2.43-2.56(\mathrm{~m}, 2 \mathrm{H})$, $2.94(\mathrm{~s}, 6 \mathrm{H}), 4.05(\mathrm{t}, J=6.8 \mathrm{~Hz}, 1 \mathrm{H}), 5.08-5.26(\mathrm{~m}, 3 \mathrm{H}), 5.77-$ $5.90(\mathrm{~m}, 1 \mathrm{H}), 6.71(\mathrm{~d}, J=8.4 \mathrm{~Hz}, 2 \mathrm{H}), 7.21-7.48(\mathrm{~m}, 6 \mathrm{H}), 7.58$ $(\mathrm{dd}, J=1.6 \mathrm{~Hz}, J=7.2 \mathrm{~Hz}, 2 \mathrm{H}) ;{ }^{13} \mathrm{C} \mathrm{NMR}\left(\mathrm{CDCl}_{3}, 100 \mathrm{MHz}\right): \delta=$ $40.01,40.49,40.49,63.25,112.42,117.52,126.77,128.45$, $128.45,128.92,131.53,132.91,134.89,150.04,166.88 ;$ IR (neat): $v=3301,3072,2978,2888,2802,1659,1652,1579$, 1524, 1455, 1353, 1227, 1189, 1164, 1132, 1062, 1027, 994, 947, 913, 815, 731, 694, 647, $540 \mathrm{~cm}^{-1}$; HRMS (ESI) calcd. for $\mathrm{C}_{19} \mathrm{H}_{24} \mathrm{~N}_{3} \mathrm{O}^{+}=[\mathrm{M}+\mathrm{H}]^{+}: \mathrm{m} / z=$ 310.1914 and for $\mathrm{C}_{19} \mathrm{H}_{23} \mathrm{~N}_{3} \mathrm{NaO}^{+}=[\mathrm{M}+\mathrm{Na}]^{+}: \mathrm{m} / z=332.1733$, found: $\mathrm{m} / z=310.1907$ (minor) and 332.1721 (major).

$\boldsymbol{N}$-(1-(pyridin-3-yl)but-3-enyl)benzohydrazide (3r; Table 2, entry 18): prepared from

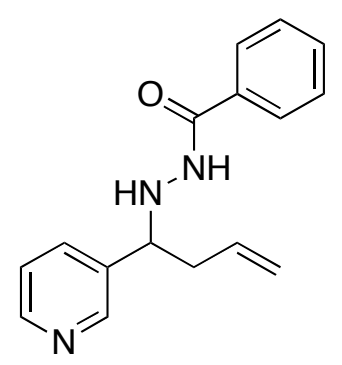

$3 r$ hydrazone $1 \mathrm{r}$ according to the general procedure for indium(I) iodidecatalyzed allylation with 2 . White solid (yield: $86 \%$ ); $\mathrm{mp}=118.4-121.6$ ${ }^{\circ} \mathrm{C} ;{ }^{1} \mathrm{H}$ NMR $\left(\mathrm{CDCl}_{3}, 400 \mathrm{MHz}\right): \delta=2.42-2.59(\mathrm{~m}, 2 \mathrm{H}), 4.22(\mathrm{t}, \mathrm{J}=6.8$ $\mathrm{Hz}, 1 \mathrm{H}), 5.12-5.24(\mathrm{~m}, 3 \mathrm{H}), 5.76-5.88(\mathrm{~m}, 1 \mathrm{H}), 7.23-7.28(\mathrm{~m}, 1 \mathrm{H}), 7.36$ (dd, $J=7.2 \mathrm{~Hz}, J=8.0 \mathrm{~Hz}, 2 \mathrm{H}), 7.46(\mathrm{dd}, J=7.2 \mathrm{~Hz}, J=8.0 \mathrm{~Hz}, 1 \mathrm{H}$ ), 7.58-7.71 (m, 4H), 8.48 (dd, $J=4.4 \mathrm{~Hz}, J=4.8 \mathrm{~Hz}, 1 \mathrm{H}), 8.59(\mathrm{~d}, J=2.4$ $\mathrm{Hz}, 1 \mathrm{H}) ;{ }^{13} \mathrm{C}$ NMR $\left(\mathrm{CDCl}_{3}, 100 \mathrm{MHz}\right): \delta=40.23,61.69,118.78,123.50$, $126.84,128.67,131.96,132.55,133.63,135.43,137.26,149.05$, 149.61, 167.63; IR (KBr): $v=3436,3019,1657,1579,1528,1459$, 1429, 1363, 1324, 1216, 1085, 1028, 928, 770, $669 \mathrm{~cm}^{-1}$; HRMS (ESI) calcd. for $\mathrm{C}_{16} \mathrm{H}_{18} \mathrm{~N}_{3} \mathrm{O}^{+}=[\mathrm{M}+\mathrm{H}]^{+}: \mathrm{m} / z=268.1444$ and for $\mathrm{C}_{16} \mathrm{H}_{17} \mathrm{~N}_{3} \mathrm{NaO}^{+}=[\mathrm{M}+\mathrm{Na}]^{+}: \mathrm{m} / z=290.1264$, found: $m / z=268.1440$ (major) and 290.1274 (minor).

Methyl 2-(benzamido)-2-methylpent-4-enoate (3u; Table 2, entry 21): prepared from

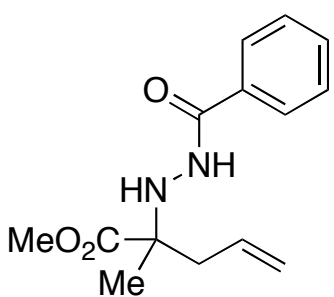

3u hydrazone $1 \mathbf{u}$ according to the general procedure for indium(I) iodidecatalyzed allylation with 2. Pale yellow oil (yield: $89 \%$ ); ${ }^{1} \mathbf{H}$ NMR $\left(\mathrm{CDCl}_{3}, 400 \mathrm{MHz}\right): \delta=1.35(\mathrm{~s}, 3 \mathrm{H}), 2.44-2.55(\mathrm{~m}, 2 \mathrm{H}), 3.71(\mathrm{~s}, 3 \mathrm{H})$, 5.03 (br s, $1 \mathrm{H}), 5.10-5.14(\mathrm{~m}, 2 \mathrm{H}), 5.73-5.83(\mathrm{~m}, 1 \mathrm{H}), 7.36-7.48(\mathrm{~m}$, $3 \mathrm{H}), 7.70(\mathrm{~d}, J=6.8 \mathrm{~Hz}, 2 \mathrm{H}), 7.97(\mathrm{br} \mathrm{s}, 1 \mathrm{H}) ;{ }^{13} \mathbf{C} \mathbf{N M R}\left(\mathrm{CDCl}_{3}\right.$, $100 \mathrm{MHz}): \delta=21.04,41.43,52.26,64.95,119.46,126.85,128.57$, 131.70, 132.10, 132.79, 166.76, 175.23; IR (neat): $v=3278,3077$, 2943, 2891, 2865, 2170, 1643, 1604, 1579, 1532, 1463, 1384, 1365, 1316, 1071, 1027, 996, 918, 883, 793, 692, $677 \mathrm{~cm}^{-1}$; HRMS (ESI) calcd. for $\mathrm{C}_{14} \mathrm{H}_{19} \mathrm{~N}_{2} \mathrm{O}_{3}{ }^{+}=$ $[\mathrm{M}+\mathrm{H}]^{+}: m / z=263.1390$, found: $m / z=263.1401$. 


\section{References}

1 Kobayashi, S.; Ogawa, C.; Konishi, H.; Sugiura, M. J. Am. Chem. Soc. 2003, 125, 6610.

2 Roush, W. R.; Adam, M. A.; Walts, A. E.; Harris, D. J. J. Am. Chem. Soc. 1986, 108, 3422.

3 Allylindium(I) has been previously reported: (a) Chan, T. H.; Yang, Y. J. Am. Chem. Soc. 1999, 121, 3228;

(b) Fontana, G.; Lubineau, A. ; Scherrmann, M.-C. Org. Biomol. Chem. 2005, 3, 1375.

4 Allylindium(III) diiodide has been previously reported: Araki, S.; Ito, H.; Katsumura, N.; Butsugan, Y. J. Organomet. Chem. 1989, 369, 291.

5 Allylindium sesquihalides have been previously reported: Araki, S.; Ito, H.; Butsugan, Y. J. Org. Chem. 1988, 53, 1833.

6 Hirabayashi, R.; Ogawa, C.; Sugiura, M.; Kobayashi, S. J. Am. Chem. Soc. 2001, 123, 9493.

7 Tan, K. L.; Jacobsen, E. N. Angew. Chem. Int. Ed. 2007, 46, 1315. 